BULL. AUSTRAL. MATH. SOC.

VOL. $28(1983), 339-342$.

\title{
A NOTE ON COMPLETE HYPERSURFACES \\ OF NON-POSITIVE RICCI CURVATURE
}

\author{
G.H. SMITH
}

In this note we point out that a recent result of Leung concerning hypersurfaces of a Euclidean space has a simple generalisation to hypersurfaces of complete simply-connected Riemannian manifolds of non-positive constant sectional curvature.

The purpose of this note is to establish the following results.

THEOREM. Let $\bar{M}$ be a complete simply-connected $n+1$ dimensional Riemannian manifold of constant curvature $C \leq 0$. Let $M$ be a complete hypersurface in $\bar{M}$ such that all sectional curvatures on $M$ are bounded away from -o. If $M$ is contained in a ball of radius $R$ then

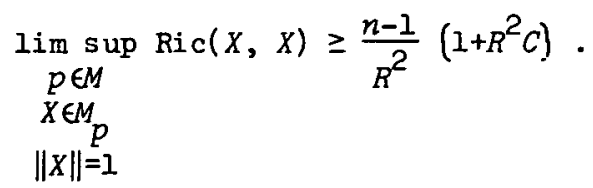

COROLLARY. Let $\bar{M}$ be as in the above theorem. Let $M$ be a complete hypersurface in $\bar{M}$ such that all sectional curvatures are bounded away from $-\infty$. If the Ricci curvature of $M$ satisfies $\operatorname{Ric}(X, X) \leq C\|X\|^{2}$ then $M$ is zonbounded.

Proof. The case $C=0$ has been proved by Leung [1]. We sketch the

Received I July 1983.

Copyright Clearance Centre, Inc. Serial-fee code: 0004-9727/83 $\$ A 2.00+0.00$ 
proof for the case $C=-1 / \rho^{2}<0$. We choose an arbitrary point $0 \in \bar{M}$, $0 \notin M$. There exists a coordinate system $r, \theta_{1}, \theta_{2}, \ldots, \theta_{n}$ on $\bar{M}-\{0\}$ and 1 - forms

$$
\begin{aligned}
\omega^{1}= & \rho d r \\
\omega^{2}= & \rho \sinh r d \theta_{1} \\
\omega^{3}= & \rho \sinh r \cos \theta_{1} d \theta_{2} \\
& \ldots \ldots \\
\omega^{n+1}= & \rho \sinh r \cos \theta_{1} \cos \theta_{2} \ldots \cos \theta_{n-1} d \theta_{n}
\end{aligned}
$$

such that the metric on $\bar{M}-\{0\}$ is given by

$$
g=\omega^{1} \otimes \omega^{1}+\omega^{2} \otimes \omega^{2}+\ldots+\omega^{n+1} \otimes \omega^{n+1} .
$$

Let $e_{1}, \ldots, e_{n+1}$ be dual to $\omega^{1}, \ldots, \omega^{n+1}$. For convenience in notation we put $\theta_{0} \equiv 0$. The connection 1 - forms for the above metric are (for $2 \leq k \leq n+1,2 \leq j<k$ )

$$
\begin{aligned}
& \omega_{1}^{k}=\cosh r \cos \theta_{0} \cos \theta_{1} \ldots \cos \theta_{k-2} d \theta_{n-1}, \\
& \omega_{j}^{k}=-\sin \theta_{j-1} \cos \theta_{j} \ldots \cos \theta_{k-2} d \theta_{k-1} .
\end{aligned}
$$

Define a vector field $X$ on $\bar{M}-\{0\}$ by

$$
x_{p}=\rho\left(r e_{1}\right)_{p} \text {. }
$$

Let

$$
V=a_{1} e_{1}+\ldots+a_{n+1} e_{n+1}
$$

be a unit vector field.

An elementary calculation shows that

$$
\begin{aligned}
\bar{D}_{V} X & =a_{1} e_{1}+\sum_{i, k} a_{i} r w_{1}^{k}\left(e_{i}\right) e_{k} \\
& =a_{1} e_{1}+r \cosh r\left(a_{2} e_{2}+\ldots+a_{n+1} e_{n+1}\right) .
\end{aligned}
$$

Cons equently, 


$$
\left\langle\bar{D}_{V} X, X\right\rangle=a_{1} r=\langle V, X\rangle
$$

and

$$
\begin{aligned}
\left\langle V, \vec{D}_{V} X\right\rangle & =a_{1}^{2}+r \cosh r\left(a_{2}^{2}+\ldots+a_{n+1}^{2}\right) \\
& \geq 1
\end{aligned}
$$

Now suppose $M$ is bounded, so that $M$ lies inside a ball of radius $R$, say. We define a function $f$ on $M$ by $f(p)=\left\langle X_{p}, X_{p}\right\rangle$ for $p \in M$. Clearly $f(p) \leq R^{2}$ for all $p \in M$ and so is bounded. We have, for a unit vector $V \in M_{p}$,

$$
\text { Hess } \begin{aligned}
f(V, V) & =V V f-D_{V} V(f) \\
& =2 V\langle V, X\rangle-2\left\langle D_{V} V, X\right\rangle \\
& =2\left\langle\bar{D}_{V} V, X\right\rangle+2\left\langle V, D_{V} X\right\rangle-2\left\langle D_{V} V, X\right\rangle \\
& \geq 2\langle B(V, V\rangle, X\rangle+2,
\end{aligned}
$$

where $B$ is the second fundamental form on $M$. Following Leung's proof we find that this inequality implies

$$
\|B(V, V)\|>\frac{1}{R}\left(1-\frac{1}{m}\right)
$$

for all positive integers $m$. Consequently $B(V, V) \neq 0$.

We now take $V_{0}$ so that $\left\|B\left(V_{0}, V_{0}\right)\right\|^{2}$ is the minimum of $\|B(V, V)\|^{2}$ for all unit vectors $V \in M_{p}$.

As shown by Leung there exists an orthonormal basis $V_{0}, Y_{1}, \ldots, Y_{n-1}$ for $M_{p}$ with $Y_{1}, \ldots, Y_{n-1}$ being an orthonormal basis for ker $B\left(V_{0}, \cdot\right)$. Hence by the Gauss equation for hypersurfaces we have 


$$
\begin{aligned}
\operatorname{Ric}\left(V_{0}, V_{0}\right) & =\sum_{i=1}^{n-1} R\left(v_{0}, y_{i}, v_{0}, y_{i}\right) \\
& =\frac{n-1}{\rho^{2}}+\sum_{i=1}^{n-1}\left\langle B\left(y_{i}, y_{i}\right), B\left(v_{0}, v_{0}\right)\right\rangle \\
& \geq \frac{1-n}{\rho^{2}}+\sum_{i=1}^{n-1}\left\|B\left(v_{0}, v_{0}\right)\right\|^{2} \\
& >\frac{1-n}{\rho^{2}}+\frac{n-1}{R^{2}}\left(1-\frac{1}{m}\right)^{2} .
\end{aligned}
$$

Hence letting $m \rightarrow \infty$ we obtain the theorem above, from which the corollary readily follows.

\section{Reference}

[1] Pui-Fai Leung, "Complete hypersurface of non-positive Ricci curvature", Bulz. Austral. Math. Soc. 27 (1983), 215-219.

School of Mathematical Sciences,

New South Wales Institute of Technology,

PO Box 123,

Broadway,

New South wales 2007,

Australia. 